\section{Validação e reprodutibilidade de sinais clínicos no diagnóstico de anemia em crianças}

\author{
Validity and reproductibility of the clinical signs \\ for the diagnosis of anemia in children
}

\author{
1 Departamento de Nutrição, \\ Universidade Federal de \\ Pernambuco, Recife, Brasil. \\ 2 Departamento de \\ Enfermagem, Universidade \\ Federal de Pernambuco, \\ Recife, Brasil. \\ Correspondência \\ M. M. Osório \\ Programa de Pós-graduação \\ em Nutrição, Departamento \\ de Nutrição, Universidade \\ Federal de Pernambuco. \\ Av. Prof. Moraes Rego s/n, \\ Cidade Universitária, \\ Recife, PE 50670-901, Brasil. \\ mosorio@ufpe.br
}

\begin{abstract}
This study aimed to assess the validity and reproducibility of clinical signs (palmar and conjunctival pallor) in the diagnosis of anemia in children 6-23 months of age in Northeast Brazil. This was a cross-sectional study with a sample of 421 children in the child care and pediatric outpatient wards at the Mother and Child Care Institute of Pernambuco. Two examiners evaluated clinical signs using the Kappa coefficient, and validation (sensitivity and specificity) was performed using hemoglobin as the standard. Clinical signs demonstrated low reproducibility (kappa 0.24-0.25). The highest sensitivity for diagnosing anemia $(\mathrm{Hb}<11 \mathrm{~g} / \mathrm{dl})$ and moderatel severe anemia $(\mathrm{Hb}<9 \mathrm{~g} / \mathrm{dl})$, respectively, was provided by combining palmar and conjuntival pallor (39.7\% and 53.5\%), followed by palmar pallor alone (29.9\% and 40.0\%). The highest specificity was provided by palmar pallor in the child as compared to the mother (95.5\% and 90.1\%, $\mathrm{Hb}<11 \mathrm{~g} / \mathrm{dl}$ and $\mathrm{Hb}<9 \mathrm{~g} / \mathrm{dl}$, respectively). Sensitivity of clinical signs was better in diagnosing moderate/severe anemia, especially when combining palmar and conjunctival pallor, suggesting that their utilization does not provide a good instrument for diagnosing mild anemia.
\end{abstract}

Diagnosis; Anemia; Child Health; Reproducibility of Results
Luciana Pedrosa Leal 1,2

Mônica Maria Osório 1

\section{Introdução}

A magnitude, a ampla distribuição espacial e os danos à saúde tornam a deficiência de ferro a mais comum desordem nutricional no mundo e os grupos de crianças pré-escolares e mulheres grávidas são os que apresentam maiores prevalências, inclusive em países desenvolvidos 1 . Estima-se uma prevalência de anemia de $39,0 \%$ entre as crianças de $0-4$ anos em países não-industrializados e de $20,1 \%$ em países industrializados 1 . Suas conseqüências como baixa imunidade, déficit no crescimento e no desenvolvimento psíquico-motor, trazem grandes preocupações às nações 2,3.

Segundo a Organização Mundial da Saúde (OMS), para o diagnóstico de anemia, a análise da hemoglobina é um exame factível em saúde pública, pelo baixo custo e simplicidade dos métodos utilizados 1 . Entretanto, algumas localidades, especialmente em países em desenvolvimento, não possuem disponibilidade para realização dos exames laboratoriais. Por conta disto, o exame físico vem sendo estudado e utilizado em países onde existem locais de difícil acesso a laboratórios e poucos recursos destinados à saúde, como uma nova forma de diagnosticar a anemia. O exame físico busca identificar a palidez em pele e mucosas como sinais clínicos indicadores da anemia. Vários segmentos anatômicos, como a conjuntiva ocular, as palmas das mãos, o leito ungueal, lábios e língua 
têm sido estudados visando a identificar aqueles que melhor diagnostiquem a anemia 4,5,6.

Todavia, o diagnóstico da anemia ferropriva em crianças, com base em sinais e sintomas, não se constitui em uma tarefa fácil 7. Uma das dificuldades em se acreditar na confiabilidade dos sinais clínicos da anemia é a reprodutibilidade dos mesmos em função da subjetividade inerente à pesquisa desses sinais 8 . Villarreal et al. 9 , em estudo realizado com crianças entre 6 e 30 meses de idade, reportam a dificuldade do uso dos sinais clínicos para diagnosticar anemia, quando destacam que a maioria das crianças com hemoglobina entre 9g/dl e 11g/dl não apresentaram sintomas e sinais clínicos de anemia.

Atualmente, em países em desenvolvimento, os profissionais de saúde vêm sendo treinados para implementar as normas de Atenção Integrada às Doenças Prevalentes da Infância (AIDPI) 10. Essas normas indicam como parâmetro para classificação da anemia e anemia grave em crianças menores de cinco anos a presença ou ausência de palidez palmar leve ou grave.

Uma vez que as normas do AIDPI 10 estão se difundindo amplamente em nossa região, realizou-se este estudo com o objetivo de avaliar a validade e reprodutibilidade dos sinais clínicos (palidez palmar e conjuntival) no diagnóstico de anemia em crianças de 6-23 meses de idade.

\section{Métodos}

O estudo foi do tipo transversal com uma amostra de 421 crianças, desenvolvido no Instituto Materno Infantil de Pernambuco (IMIP), situado na Cidade do Recife, Pernambuco, Brasil. Os locais para coleta de dados foram os ambulatórios de puericultura e pediatria.

O cálculo do tamanho da amostra baseouse na fórmula para avaliar a sensibilidade e especificidade de testes diagnósticos 11: $\mathrm{N}=\mathrm{Z} \times \mathrm{Z}$ $(\mathrm{P}(1-\mathrm{P})) /(\mathrm{D} / \mathrm{D})$.

Para o valor de $\mathrm{P}$, tomou-se como base a maior sensibilidade $(49,1 \%)$ do estudo de Sdepanian et al. 8 para o diagnóstico da anemia avaliado pela presença de palidez palmar e conjuntival. O valor de D (semi-amplitude do intervalo de confiança) foi definido como igual a $5 \%$ e o de Z, como 1,96 (para $\alpha=0,05$ e IC = 95\%). Com um acréscimo de $10 \%$ para compensar as perdas, seria necessária uma amostra de 420 crianças.

A seleção da amostra teve como critério de inclusão as crianças de 6 a 23 meses de idade, acompanhadas por mãe biológica às consultas nos ambulatórios de puericultura e de pediatria. A captação das crianças durante o trabalho de campo foi realizada diariamente, independente do motivo da consulta, até alcançar o número previsto da amostra. Como critério de exclusão, consideraram-se as crianças portadoras de doenças crônicas (cardiopatias, doenças reumáticas, nefropatias, afecções do aparelho gastrointestinal, doenças respiratórias etc), com diagnóstico confirmado no momento da consulta.

Os sinais clínicos foram avaliados por dois observadores, sendo uma enfermeira e uma médica pediatra, previamente treinadas pelas normas do AIDPI 10, por meio da avaliação clínica da palidez palmar e da conjuntiva ocular da criança sob iluminação artificial (lâmpada fluorescente). Cada um dos examinadores, em momentos diferentes, realizou a observação da palma da mão e da conjuntiva ocular da criança.

A avaliação clínica da palidez palmar foi baseada na experiência e julgamento de cada examinador de acordo com o que orienta as normas do AIDPI 10. Para avaliar a palidez palmar, o examinador abria uma das mãos da criança, estendendo parcialmente os dedos e observava a coloração da palma. Em seguida, o examinador realizava a mesma observação utilizando a palma da mão da mãe biológica como padrão para identificar palidez palmar.

A avaliação clínica da palidez conjuntival foi realizada baseando-se no julgamento dos examinadores acerca da coloração da conjuntiva ocular das crianças, utilizando o conceito de descoramento das mucosas como sendo a diminuição ou a perda da cor róseo-avermelhada, considerada como normal 12 . Para a avaliação da palidez conjuntival, o examinador evertia a pálpebra inferior e observava diretamente a coloração.

O projeto foi aprovado pelo Comitê de Ética e Pesquisa em Seres Humanos do Comitê Nacional de Ética. Os exames laboratoriais e clínicos das crianças foram realizados após consentimento por escrito fornecido por suas mães. Todas as crianças e genitoras, diagnosticadas como anêmicas pela pesquisa, receberam tratamento com sulfato ferroso oral.

\section{Análise dos dados}

Os dados coletados foram processados em microcomputador, utilizando-se o programa Epi Info versão 6.04. Os diagnósticos de anemia foram baseados nos padrões da OMS, que considera como anêmicas as crianças de 6 a 59 meses de idade, com valor de hemoglobina abaixo de $11 \mathrm{~g} / \mathrm{dl} 1$ e como anêmicas moderadas/gra- 
ves, as crianças com hemoglobina menor que $9 \mathrm{~g} / \mathrm{dl} 7$.

A reprodutibilidade foi avaliada usando-se o cálculo da concordância global (Po), concordância em diagnósticos positivos - $\mathrm{Po}(+)$ e negativos - Po(-), e o cálculo do coeficiente de kappa (k), que demonstra a concordância ajustada, levando em consideração a concordância devido à chance entre os examinadores. Sua escala propõe os seguintes valores $11:<0,00=$ ruim; 0,00-0,20 = fraca; $0,21-0,40=$ sofrível; $0,41-0,60=$ regular; $0,61-0,80=$ boa; $0,81-0,99=$ ótima; $1=$ perfeita. A validação (sensibilidade, especificidade, valor preditivo positivo - VPP e valor preditivo negativo - VPN) foi realizada utilizandose o valor da hemoglobina como padrão. A concentração sangüínea de hemoglobina foi determinada mediante coleta de sangue venoso, em contador de células automático, marca Coulter, do tipo T-890. A análise da concentração de hemoglobina somente foi realizada após a etapa de avaliação dos sinais clínicos pelos examinadores, e as genitoras não foram informadas sobre a impressão diagnóstica dos mesmos.

\section{Resultados}

Para verificar a prevalência de anemia foi realizada a análise de hemoglobina nas crianças e em suas respectivas mães, exceto em 19 crianças por dificuldade de punção e/ou não aceitação da mãe, e em 4 mães por recusa na realização do exame. Também foram excluídas 11 mães que se encontravam grávidas.
A prevalência de anemia nas crianças foi de $89,1 \%$, com uma média de hemoglobina de 9,8 $\pm 1 \mathrm{~g} / \mathrm{dl}$ e nas mães foi de $45,3 \%$, com uma média de hemoglobina de $12 \pm 1 \mathrm{~g} / \mathrm{dl}$. A prevalência de anemia moderada/grave nas crianças foi de $19,9 \%$. A mediana de idade das crianças foi de 8 meses, estando 68,9\% entre 6 - 11 meses, $22,6 \%$ entre $12-17$ meses e apenas $8,6 \%$ entre 18-23 meses. A distribuição entre os sexos foi de $49,9 \%$ para o sexo feminino e $50,1 \%$ para o masculino.

\section{Avaliação da concordância interexaminadores}

A Tabela 1 mostra a relação dos sinais clínicos com base na observação dos examinadores I e II. A concordância, calculada pelo coeficiente de kappa, foi considerada sofrível para a palidez palmar e de conjuntiva ocular individualmente, para a palidez palmar identificada utilizando-se a palma da mão da mãe como padrão e para a combinação da palidez palmar ou palidez de conjuntiva ocular, individualmente ou juntas.

Em todos os sinais clínicos, a concordância em diagnósticos negativos foi maior do que a concordância em diagnósticos positivos. Entre os sinais clínicos, a combinação da palidez palmar ou conjuntival apresentou maior concordância em diagnósticos positivos. A concordância global foi igual ou maior a 0,70 incluindo todos os sinais clínicos, exceto a combinação da palidez palmar ou de conjuntiva.

Para a avaliação da reprodutibilidade nas crianças com anemia moderada/grave, o coefi-

Concordância entre os examinadores I e II na identificação dos sinais clínicos.

\begin{tabular}{|c|c|c|c|c|}
\hline Sinal clínico & $\begin{array}{l}\text { Coeficiente } \\
\text { de kappa }\end{array}$ & IC95\% & Concordâncias & IC95\% \\
\hline Palidez palmar & 0,25 (sofrível) & $\begin{array}{l}0,12-0,40 \\
0,36-0,55 \\
0,74-0,84\end{array}$ & $\begin{array}{c}\mathrm{Po}=0,70 \\
\mathrm{Po}(+)=0,45 \\
\mathrm{Po}(-)=0,80\end{array}$ & $0,66-0,75$ \\
\hline $\begin{array}{l}\text { Palidez palmar comparando } \\
\text { com a palma da mão da mãe }\end{array}$ & 0,25 (sofrível) & $\begin{array}{l}0,11-0,44 \\
0,25-0,48 \\
0,82-0,90\end{array}$ & $\begin{array}{c}\mathrm{Po}=0,78 \\
\mathrm{Po}(+)=0,36 \\
\mathrm{Po}(-)=0,86\end{array}$ & $0,73-0,82$ \\
\hline Palidez de conjuntiva & 0,24 (sofrível) & $\begin{array}{l}0,11-0,43 \\
0,26-0,51 \\
0,81-0,90\end{array}$ & $\begin{array}{c}\mathrm{Po}=0,77 \\
\mathrm{Po}(+)=0,38 \\
\mathrm{Po}(-)=0,86\end{array}$ & $0,72-0,81$ \\
\hline Palidez palmar ou de conjuntiva & 0,24 (sofrível) & $\begin{array}{l}0,12-0,38 \\
0,41-0,59 \\
0,68-0,80\end{array}$ & $\begin{array}{c}\mathrm{Po}=0,66 \\
\mathrm{Po}(+)=0,50 \\
\mathrm{Po}(-)=0,74\end{array}$ & $0,61-0,71$ \\
\hline
\end{tabular}


ciente de kappa foi considerado sofrível para a palidez palmar, palidez palmar comparada com a palma da mãe e palidez conjuntival (Tabela 2). A concordância em diagnósticos positivos foi maior para a combinação da palidez palmar ou conjuntival, embora o coeficiente de kappa tenha sido considerado fraco.

Sensibilidade, especificidade, VPP e VPN

dos sinais clínicos para o diagnóstico

de anemia em crianças, utilizando o ponto

de corte da hemoglobina de $11 \mathrm{~g} / \mathrm{dl}$

De acordo com os examinadores I e II, a sensibilidade foi maior para a combinação da palidez palmar ou de conjuntiva, seguida pela palidez palmar isoladamente. As maiores especificidades foram para a palidez palmar, utilizando a palma da mão da mãe como padrão e para a palidez de conjuntiva (Tabela 3 ).

Os VPP situaram-se acima de $90,0 \%$ para todos os sinais, sendo os maiores valores para a palidez de conjuntiva e para a palidez palmar comparando com a palma da mão da mãe.

Sensibilidade, especificidade, VPP e VPN dos sinais clínicos utilizando o ponto de corte de $9 \mathrm{~g} / \mathrm{dl}$

As maiores sensibilidades no diagnóstico da anemia moderada/grave, segundo os examinadores I e II, foram com o uso da combinação da palidez palmar ou da conjuntiva e da palidez palmar isoladamente (Tabela 4 ).
A especificidade apresentou maiores valores para a palidez palmar comparada com a palma da mão da mãe e para a palidez de conjuntiva.

Os maiores VPP foram para a palidez de conjuntiva segundo o examinador I e para a palidez palmar comparando com a palma da mão da mãe segundo o examinador II.

\section{Discussão}

As crianças de 6-23 meses estudadas apresentaram uma alta prevalência de anemia $(89,1 \%)$, estando a grande maioria com anemia leve e apenas $19,9 \%$ apresentaram anemia moderada/grave.

Em estudo realizado no Estado de Pernambuco, foi encontrada uma prevalência de anemia entre crianças de 6-59 meses de 40,9\%, apresentando uma prevalência de $39,6 \%$ na região metropolitana do Recife, sendo de $61,8 \%$ e $31,0 \%$ nas crianças de 6-23 e 24-59 meses, respectivamente 13 . Segundo a OMS, a maior prevalência de deficiência de ferro em menores de cinco anos ocorre durante o segundo ano de vida, devido ao baixo conteúdo de ferro nas dietas e o rápido crescimento durante o primeiro ano de vida 1.

Este estudo revelou uma prevalência de anemia nas mães de $45,3 \%$, semelhante à prevalência estimada para mulheres em idade reprodutiva em países em desenvolvimento pela OMS, que foi de $42,3 \% 1$.

\begin{tabular}{|c|c|c|c|c|}
\hline Sinal clínico & $\begin{array}{l}\text { Coeficiente } \\
\text { de kappa }\end{array}$ & IC95\% & Concordâncias & IC95\% \\
\hline Palidez palmar & 0,22 (sofrível) & $\begin{array}{l}0,11-0,36 \\
0,35-0,71 \\
0,54-0,81\end{array}$ & $\begin{array}{c}\mathrm{Po}=0,63 \\
\mathrm{Po}(+)=0,52 \\
\mathrm{Po}(-)=0,69\end{array}$ & $0,51-0,73$ \\
\hline $\begin{array}{l}\text { Palidez palmar comparando } \\
\text { com a palma da mão da mãe }\end{array}$ & 0,26 (sofrível) & $\begin{array}{l}0,12-0,41 \\
0,21-0,66 \\
0,68-0,90\end{array}$ & $\begin{array}{c}\mathrm{Po}=0,71 \\
\mathrm{Po}(+)=0,42 \\
\mathrm{Po}(-)=0,81\end{array}$ & $0,60-0,81$ \\
\hline Palidez de conjuntiva & 0,27 (sofrível) & $\begin{array}{l}0,15-0,43 \\
0,27-0,73 \\
0,62-0,88\end{array}$ & $\begin{array}{c}\mathrm{Po}=0,69 \\
\mathrm{Po}(+)=0,49 \\
\mathrm{Po}(-)=0,78\end{array}$ & $0,56-0,80$ \\
\hline Palidez palmar ou de conjuntiva & 0,11 (fraco) & $\begin{array}{l}0,04-0,23 \\
0,36-0,72 \\
0,36-0,72\end{array}$ & $\begin{array}{c}\mathrm{Po}=0,55 \\
\mathrm{Po}(+)=0,55 \\
\mathrm{Po}(-)=0,55\end{array}$ & $0,42-0,67$ \\
\hline
\end{tabular}


Sensibilidade, especificidade, valores preditivos positivos e negativos dos sinais clínicos no diagnóstico de anemia em crianças, utilizando a hemoglobina como padrão ouro, segundo os examinadores I e II.

\begin{tabular}{|c|c|c|c|c|c|c|c|c|}
\hline \multirow[t]{2}{*}{ Sinal clínico } & \multicolumn{2}{|c|}{ Sensibilidade } & \multicolumn{2}{|c|}{ Especificidade } & \multicolumn{2}{|c|}{ VPP } & \multicolumn{2}{|c|}{ VPN } \\
\hline & $\%$ & IC95\% & $\%$ & IC95\% & $\%$ & IC95\% & $\%$ & IC95\% \\
\hline \multicolumn{9}{|l|}{ Examinador I } \\
\hline Palidez palmar & 29,9 & $25,2-35,0$ & 75,0 & $59,4-86,3$ & 90,7 & $83,6-95,0$ & 11,6 & $8,2-16,1$ \\
\hline $\begin{array}{l}\text { Palidez palmar comparando } \\
\text { com a palma da mão da mãe }\end{array}$ & 25,4 & $21,1-30,3$ & 81,8 & $66,8-91,3$ & 91,9 & $84,2-96,2$ & 11,9 & $8,6-16,2$ \\
\hline Palidez de conjuntiva & 17,4 & $13,6-21,9$ & 90,2 & $75,9-96,8$ & 93,7 & $83,7-97,9$ & 11,6 & $8,4-15,8$ \\
\hline Palidez palmar ou de conjuntiva & 39,7 & $34,5-45,1$ & 70,7 & $54,3-83,4$ & 91,8 & $85,9-95,5$ & 12,4 & $8,6-17,5$ \\
\hline \multicolumn{9}{|l|}{ Examinador II } \\
\hline Palidez palmar & 28,0 & $23,4-33,0$ & 86,4 & $72,0-94,3$ & 94,2 & $87,2-97,6$ & 13,2 & $9,6-17,8$ \\
\hline $\begin{array}{l}\text { Palidez palmar comparando } \\
\text { com a palma da mão da mãe }\end{array}$ & 12,1 & $9,0-16,2$ & 95,5 & $83,3-99,2$ & 95,5 & $83,3-99,2$ & 12,1 & $9,0-16,2$ \\
\hline Palidez de conjuntiva & 21,1 & $16,8-26,2$ & 91,9 & $77,0-97,9$ & 95,6 & $86,8-98,9$ & 12,3 & $8,8-16,9$ \\
\hline Palidez palmar ou de conjuntiva & 32,5 & $27,3-38,1$ & 81,1 & $64,3-91,4$ & 93,5 & $86,5-97,1$ & 12,6 & $8,8-17,7$ \\
\hline
\end{tabular}

VPP = valores preditivos positivos; VPN = valores preditivos negativos.

Sensibilidade, especificidade, valores preditivos positivos e negativos dos sinais clínicos no diagnóstico de anemia moderada/grave em crianças, utilizando a hemoglobina como padrão ouro, segundo os examinadores I e II.

\begin{tabular}{|c|c|c|c|c|c|c|c|c|}
\hline \multirow[t]{2}{*}{ Sinal clínico } & \multicolumn{2}{|c|}{ Sensibilidade } & \multicolumn{2}{|c|}{ Especificidade } & \multicolumn{2}{|c|}{ VPP } & \multicolumn{2}{|c|}{ VPN } \\
\hline & $\%$ & IC95\% & $\%$ & IC95\% & $\%$ & IC95\% & $\%$ & IC95\% \\
\hline \multicolumn{9}{|l|}{ Examinador I } \\
\hline Palidez palmar & 40,0 & $29,4-51,6$ & 73,3 & $68,0-78,0$ & 27,1 & $19,5-36,2$ & 83,1 & $78,1-87,2$ \\
\hline $\begin{array}{l}\text { Palidez palmar comparando } \\
\text { com a palma da mão da mãe }\end{array}$ & 31,3 & $21,6-42,7$ & 77,0 & $72,0-81,4$ & 25,3 & $17,3-35,2$ & 81,8 & $76,9-85,9$ \\
\hline Palidez de conjuntiva & 26,8 & $17,3-38,8$ & 85,8 & $81,3-89,4$ & 30,2 & $19,6-43,2$ & 83,6 & $79,0-87,4$ \\
\hline Palidez palmar ou de conjuntiva & 53,5 & $41,4-65,3$ & 64,8 & $59,2-70,1$ & 25,9 & $19,1-33,8$ & 85,9 & $80,6-90,0$ \\
\hline \multicolumn{9}{|l|}{ Examinador II } \\
\hline Palidez palmar & 38,5 & $27,9-50,2$ & 76,7 & $71,5-81,2$ & 29,1 & $20,8-39,0$ & 83,3 & $78,4-87,3$ \\
\hline $\begin{array}{l}\text { Palidez palmar comparando } \\
\text { com a palma da mão da mãe }\end{array}$ & 16,9 & $9,6-27,5$ & 90,1 & $86,1-93,1$ & 29,5 & $17,2-45,4$ & 81,5 & $76,9-85,4$ \\
\hline Palidez de conjuntiva & 27,7 & $17,7-40,4$ & 82,1 & $77,0-86,3$ & 26,5 & $16,8-38,8$ & 83,0 & $78,0-87,2$ \\
\hline Palidez palmar ou de conjuntiva & 43,1 & $31,1-55,9$ & 71,8 & $66,1-76,9$ & 26,2 & $18,4-35,7$ & 84,5 & $79,1-88,7$ \\
\hline
\end{tabular}

VPP = valores preditivos positivos; VPN = valores preditivos negativos.

\section{Reprodutibilidade dos sinais clínicos}

Para os sinais clínicos, a reprodutibilidade foi considerada sofrível (kappa de 0,24-0,25). Este resultado corrobora com outros estudos onde a concordância encontrada variou de sofrível a regular 14,15,16,17,18.

Os sinais clínicos apresentaram uma concordância em diagnósticos negativos (ausência de palidez) maior que em diagnósticos positivos para identificar palidez, como demonstrado também em outros estudos na literatura 8,19.

Entre os sinais clínicos, a maior concordância em diagnósticos positivos ocorreu quando se analisou a presença da combinação da palidez palmar ou de conjuntiva ocular. Sdepanian et al. 8, em estudo realizado em crianças de 672 meses observaram também que a reprodu- 
tibilidade dos sinais clínicos se eleva quanto maior o número de sinais positivos.

Quando selecionadas apenas as crianças com anemia moderada/grave, não foi observada melhora na concordância entre os dois examinadores na identificação da palidez. Alguns autores, no entanto, referem que a concordância tende a melhorar quanto maior a gravidade da anemia 8,19 .

Salienta-se que a concordância entre examinadores nem sempre reflete a veracidade no diagnóstico, pois a igualdade nos diagnósticos não implica que o mesmo esteja correto. Luby et al. 20 referem que a validade dos sinais clínicos é o mais importante critério para diagnosticar anemia e o considera suficiente para diagnosticar anemias moderada e severa.

\section{Diagnóstico da anemia mediante os sinais clínicos}

Segundo a OMS, o exame clínico da palidez de pele e mucosas pode ser usado para detectar anemia severa em crianças 1,7. O sinal da palidez palmar para identificar anemia vem sendo utilizado pelas normas do AIDPI 10, com o intuito de identificar as crianças de risco e referenciá-las a serviços especializados ou tratar aquelas com menor gravidade, na própria comunidade 10 .

Neste estudo, o uso dos sinais clínicos para diagnosticar anemia demonstrou uma baixa sensibilidade. Entre os sinais avaliados isoladamente, a palidez palmar foi o que identificou um maior número de crianças anêmicas segundo os dois examinadores, corroborando com os estudos de Luby et al. 20 e Weber et al. 16. A sensibilidade aumentou quando se avaliou a presença da combinação da palidez palmar ou de conjuntiva ocular. Kalter et al. 5 referem que a palidez de conjuntiva ou a palidez palmar combinada pode melhorar o diagnóstico de anemia moderada ou leve, considerando como ponto de corte a hemoglobina entre $5 \mathrm{~g} / \mathrm{dl}$ e $10 \mathrm{~g} / \mathrm{dl}$. Outros trabalhos também têm sugerido que a associação da palidez em vários locais conjuntamente seria uma forma de melhorar o desempenho dos sinais clínicos no diagnóstico de anemia 6,8,14,20.

A ausência de palidez, por sua vez, não indica, necessariamente, a ausência de anemia, sendo identificado neste estudo um grande número de falsos-negativos. Isso implica que muitas crianças diagnosticadas como não anêmicas eram na realidade anêmicas. Os sinais e sintomas da anemia, principalmente a anemia leve, geralmente passam despercebidos, e algumas pessoas aparentemente normais estão anê- micas 11,21. Para efeito prático, o alto número de falsos-negativos implicaria em crianças anêmicas, pelo padrão da OMS 1 , sendo privadas de tratamento que combata ou elimine a anemia, principalmente em nosso meio onde a prevalência de anemia é elevada.

A palidez palmar diagnosticada quando comparada a palma da mão da criança com a palma da mão da mãe, como uma das alternativas propostas pelo AIDPI 10, não parece ser um bom indicador para determinar anemia em crianças, uma vez que as mulheres em idade reprodutiva também se constituem em um grupo de risco para anemia. Isto pode ser observado nesta amostra, onde $45,3 \%$ das mães eram anêmicas, possibilitando um maior diagnóstico de falso-negativo.

Ao analisar os sinais isoladamente, outros estudos têm feito referência à palidez de conjuntiva e/ou leito ungueal como locais com melhores resultados em relação ao diagnóstico de anemia e anemia grave 5,15,22. Segundo a OMS 1 , para crianças jovens, a palidez palmar é preferível à palidez de conjuntiva ocular, devido à freqüência de conjuntivites, que podem alterar a coloração da mucosa em crianças anêmicas.

Ao utilizar o ponto de corte de $9 \mathrm{~g} / \mathrm{dl}$, houve um aumento da sensibilidade de todos os sinais clínicos, contudo, permanecendo com valores baixos, o que não é o esperado de um bom teste diagnóstico.

Houve um grande número de falsos-positivos (de $69,0 \%$ a $74,0 \%$ das crianças consideradas anêmicas pelos sinais clínicos), ou seja, muitas crianças não anêmicas ou com anemia leve foram diagnosticadas como tendo anemia moderada ou grave. Neste caso, um possível tratamento não caracterizaria um problema, visto que foi alta a prevalência encontrada de anemia leve.

Esses resultados sugerem que os sinais clínicos apresentam uma melhor sensibilidade quanto maior o grau de anemia. Sabendo-se que a palidez palmar ou conjuntival se torna menos visível na anemia leve, entende-se, dessa maneira, a dificuldade em identificar as verdadeiras crianças doentes. Por outro lado, ao diminuir o ponto de corte para identificar crianças com anemia moderada ou grave, nas quais a palidez torna-se mais evidente, a sensibilidade do teste aumenta.

O VPP foi alto para todos os sinais clínicos, estando acima de 90,0\%. Em contrapartida, o VPN foi baixo (abaixo de 14,0\%). É importante referir que os valores preditivos dependem da sensibilidade e especificidade do teste em questão e da prevalência da doença na população, e no caso deste estudo, a prevalência encontrada 
foi muito alta. Portanto, um VPP alto refere-se à boa capacidade do teste em identificar os verdadeiros doentes entre aqueles diagnosticados como doentes pelo mesmo, o que somente é possível afirmar para a população aqui estudada. Por outro lado, ao utilizar o ponto de corte da hemoglobina de $9 \mathrm{~g} / \mathrm{dl}$, a prevalência da anemia diminui e conseqüentemente há um aumento dos VPN e diminuição dos VPP.

Outros estudos também mostraram que a palidez foi melhor para diagnosticar a anemia severa ou moderada do que a anemia leve 6,20,23. No entanto, neste estudo praticamente não foram encontradas crianças com anemia grave e, portanto, apenas pode-se inferir uma melhor validade em diagnosticar anemia moderada.

Percebe-se que a utilização dos sinais clínicos não se constitui em um bom instrumento para diagnosticar anemia leve 6,8,20,22,23. Os dados aqui apresentados mostraram uma baixa sensibilidade e reprodutibilidade dos sinais avaliados (palidez palmar e conjuntival). Por outro lado, a sensibilidade melhorou no diagnóstico da anemia moderada/grave, reduzindo o número de falsos-negativos, fato importante quando, diante de prevalências altas de anemia, pode-se intervir no tratamento e/ou encaminhamento dessas crianças a serviços especializados.

\section{Resumo}

Este trabalho teve como objetivo avaliar a validade e reprodutibilidade dos sinais clínicos (palidez palmar e conjuntival) no diagnóstico de anemia em crianças de 623 meses, no Nordeste do Brasil, por meio de estudo transversal com amostra de 421 crianças, realizado nos ambulatórios de pediatria e puericultura do Instituto Materno Infantil de Pernambuco. Os sinais clínicos foram avaliados por dois examinadores. A reprodutibilidade foi avaliada pelo coeficiente de kappa e a validação (sensibilidade e especificidade) foi realizada utilizandose a hemoglobina como padrão. Os sinais clínicos demonstraram baixa reprodutibilidade (kappa de 0,24$0,25)$. A maior sensibilidade para diagnosticar anemia $(\mathrm{Hb}<11 \mathrm{~g} / \mathrm{dl})$ e anemia moderadal grave $(\mathrm{Hb}<9 \mathrm{~g} / \mathrm{dl})$, respectivamente, foi apresentada pela combinação de palidez palmar ou conjuntival (39,7\% e 53,5\%), seguida pela palidez palmar isoladamente (29,9\% e 40,0\%). A maior especificidade foi para a palidez palmar comparada à palma da mão da mãe (95,5\% e 90,1\% para $\mathrm{Hb}<11$ g/dle $\mathrm{Hb}<9 \mathrm{~g} / \mathrm{dl}$, respectivamente). A sensibilidade dos sinais clínicos foi melhor para diagnosticar a anemia moderada/grave, especialmente quando a palidez palmar e conjuntival são combinadas, sugerindo que a utilização dos mesmos não se constitui em um bom instrumento para diagnosticar anemia leve.

Diagnóstico; Anemia; Saúde Infantil; Reprodutibilidade de Resultados
Assim, o uso dos sinais clínicos no diagnóstico de anemia tem sido relatado por estudos realizados em países da África como um método possível 5,6,16,17,20 especialmente pela alta prevalência de anemia moderada/grave encontrada nesses locais. Em virtude dessas áreas serem de altas prevalências de malária, geralmente esta infecção está associada a uma anemia mais grave, sendo esta uma das principais complicações em crianças de seis meses a dois anos de idade. Entretanto, a anemia grave não parece ter altas prevalências em nosso país. Estudo realizado no Estado de Pernambuco, Nordeste do Brasil, onde a prevalência de anemia encontrada em crianças de 6-59 meses foi de 40,9\%, a prevalência da anemia moderada foi de $10,6 \%$ no interior rural (a área mais carente do estado) e de 6,0\% nas demais áreas geográficas; e a prevalência mais alta de anemia severa foi de $1,3 \%$ no interior rural 13 . Na Cidade de São Paulo, Monteiro \& Szarfarc 24, também demonstraram uma prevalência de anemia severa $(<9,5 \mathrm{~g} / \mathrm{dl})$ menor do que os outros graus de anemia. Sendo assim, as maiores prevalências no Brasil parecem ser de anemia leve, que possivelmente está mais determinada por dificuldades de acesso a uma alimentação rica em ferro, não sugerindo a adoção do exame clínico da palidez de pele e mucosas como único meio para diagnosticar anemia.

\section{Colaboradores}

L. P. Leal e M. M. Osório participaram da concepção da pesquisa, elaboração dos instrumentos de coleta dos dados, revisão da literatura, análise e processamento dos dados e redação do artigo.

\section{Agradecimentos}

Os autores agradecem à Organização Pan-Americana da Saúde/Ministério da Saúde, Brasil, pelo suporte financeiro e ao Instituto Materno Infantil de Pernambuco (IMIP) pelo apoio na operacionalização desta pesquisa. Os agradecimentos se estendem às mães $\mathrm{e}$ crianças participantes do estudo. 


\section{Referências}

1. World Health Organization/United Nations Children's Fund/United Nations University. Iron deficiency anaemia. Assessment, prevention and control. A guide for programme managers. Geneva: World Health Organization/United Nations Children's Fund/United Nations University; 2001.

2. Pollit E. Iron deficiency anemia and later mental retardation. Am J Clin Nutr 1999; 69:4-5.

3. Lozoff B, Jimenez E, Wolf A. Long term developmental outcome of infants with iron deficiency. N Engl J Med 1991; 325:687-94.

4. Strobach RS, Anderson SK, Doll DC, Ringenberg QS. The value of the physical examination in the diagnosis of anemia. Arch Intern Med 1988; 148: 831-2.

5. Kalter HD, Burnhan G, Kolstad PR, Hossain M, Schillinger JA, Khan NZ, et al. Evaluation of clinical signs to diagnose anaemia in Uganda and Bangladesh, in areas with and without malaria. Bull World Health Organ 1997; 75 Suppl 1:103-11.

6. Stoltzfus RJ, Edward-Raj A, Dreyfuss ML, Albonico M, Montresor A, Thapa MD, et al. Clinical pallor is useful to detect severe anemia in populations where anemia is prevalent and severe. J Nutr 1999; 129:1675-81.

7. Demaeyer E. Preventing and controlling iron deficiency anaemia through primary health care. A guide for health administrators and programme managers. Geneva: World Health Organization; 1989.

8. Sdepanian VL, Silvestrini WS, Morais MB. Limitação diagnóstica do exame físico na identificação de crianças com anemia. Rev Assoc Med Bras 1996; 42:169-74.

9. Villarreal DM, Rocco R, Gaviña ME, Bailatti N. Inadecuada prescripción de hierro como factor de riesgo de anemia ferropenica pediatrica. Revista Medicina Infantil 1999; 6:115-9.

10. Ministério da Saúde/Organização Pan-Americana da Saúde/Organização Mundial da Saúde. Atenção integrada às doenças prevalentes na infância: avaliar e classificar a criança doente de 2 meses a 5 anos de idade. Brasília: Ministério da Saúde/Organização Pan-Americana da Saúde/Organização Mundial da Saúde; 2003.

11. Andrade ALS, Zicker F. Métodos de investigação epidemiológica em doenças transmissíveis. Brasília: Organização Pan-Americana da Saúde; 1997.

12. Porto CC. Exame clínico. Rio de Janeiro: Guanabara Koogan; 1992.
13. Osório MM, Lira PIC, Batista-Filho M, Ashworth A. Prevalence of anemia in children 6-59 months old in the State of Pernambuco, Brazil. Rev Panam Salud Publica 2001; 10:101-7.

14. Nardone DA, Roth KM, Mazur DJ, McAfee JH. Usefulness of physical examination in detecting the presence or absence of anemia. Arch Intern Med 1990; 150:201-4.

15. Zucker JR, Perkins BA, Jafari H, Otieno J, Obonyo C, Campbell CC. Clinical signs for the recognition of children with moderate or severe anaemia in Western Kenya. Bull World Health Organ 1997; 75 Suppl 1:97-102.

16. Weber MW, Kellingray SD, Palmer A, Jaffar S, Mulholland EK, Greenwood BM. Pallor as a clinical sign of severe anaemia in children: an investigation in the Gambia. Bull World Health Organ 1997; 75 Suppl 1:113-8.

17. Muhe L, Oljira B, Degefu H, Jaffar S, Weber MW. Evaluation of clinical pallor in the identification and treatment of children with moderate and severe anaemia. Trop Med Int Health 2000; 5:805-10.

18. Kahigwa E, Schellenberg D, Schellenberg JA, Aponte JJ, Alonso PL, Menendez C. Inter-observer variation in the assessment of clinical signs in sick Tanzanian children. Trans R Soc Trop Med Hyg 2002; 96:162-6.

19. Gjorup T, Bugge PM, Hendriksen C, Jensen AM. A critical evaluation of the clinical diagnosis of anemia. Am J Epidemiol 1986; 124:657-65.

20. Luby SP, Kazembe PN, Redd SC, Ziba C, Nwanyanwu OC, Hightower AW, et al. Using clinical signs to diagnose anaemia in african children. Bull World Health Organ 1995; 73 Suppl 4:477-82.

21. Torres AF. Diagnóstico de anemia: un alerta para los profesionales de la salud. Adolesc Latinoam 1999; 1:231-41.

22. Spinelli MGN, Souza JMP, Souza SB, Sesoko EH. Confiabilidade e validade da palidez palmar e de conjuntivas como triagem de anemia. Rev Saúde Pública 2003; 37:404-8.

23. Montressor A, Albonico M, Khalfan N, Stoltzfus RJ, Tielsch JM, Chwaya HM, et al. Field trial of a haemoglobin colour scale: an effective tool to detect anaemia in preschool children. Trop Med Int Health 2000; 5:129-33.

24. Monteiro CA, Szarfarc SC. Estudo das condições de saúde das crianças no município de São Paulo, SP (Brasil), 1984-1985. Rev Saúde Pública 1987; 21:255-60.

Recebido em 08/Jun/2004

Versão final reapresentada em 22/Out/2004

Aprovado em 10/Nov/2004 